\title{
Metastatic lobular carcinoma of the breast masquerading as a primary rectal cancer
} Ikuo Matsuda ${ }^{1}$, Nagahide Matsubara², Nobuo Aoyama ${ }^{3}$, Mie Hamanaka², Daisuke Yamagishi ${ }^{2}$, Takashi Kuno $^{2}$,
Kiyoshi Tsukamoto ${ }^{2}$, Tomoki Yamano ${ }^{2}$, Masafumi Noda ${ }^{2}$, Hiroki Ikeuchi ${ }^{2}$ Naohiro Tomita ${ }^{2}$ and Seiichi Hirota ${ }^{2}$

\begin{abstract}
Background: Colorectal metastasis of lobular carcinoma of the breast is a diagnostic challenge. It may macroscopically simulate primary colon cancer or inflammatory bowel disease. In some cases, the interval between the primary breast cancer and metastatic colorectal lesions is so long that the critical records for diagnosis including history might be lost or missed.

Case presentation: Reported herein is a case of metastatic lobular carcinoma of the breast masquerading as a primary rectal cancer developed in a 62-year-old Japanese woman. The case initially presented as a circumferential rectal lesion, and information on the patient's history of breast cancer was not noted. As the result of endoscopic biopsy, diagnosis of poorly differentiated rectal adenocarcinoma was made. The lesion was surgically resected after chemo-radiotherapy. Histopathological examination of the resected specimen with hematoxylin and eosin (HE) stain revealed a single-file arrangement of the tumor cells, reminiscent of lobular carcinoma of the breast. Immunohistochemical analysis revealed an immunophenotype consistent with lobular carcinoma of the breast. Because further review of the patient's history revealed an occurrence of 'poorly differentiated adenocarcinoma of the breast', which she had experienced 24 years earlier, the final diagnosis of the lesion was made as rectal metastasis from lobular breast carcinoma.

Conclusions: Poorly differentiated adenocarcinoma of the colorectum is rarer than that of the stomach. Linitis plastica-type cancer of the colorectum is also rarer than that of the stomach. A lesson from the present case is that before we conclude a linitis plastica-type cancer of poorly differentiated type as a primary colorectal cancer, it is critical to exclude a possibility of metastatic colorectal cancer.
\end{abstract}

Keywords: Lobular carcinoma, Breast, Rectal metastasis

\section{Background}

Colorectal metastasis of lobular carcinoma of the breast is a diagnostic challenge. It macroscopically simulates primary colon cancer or inflammatory bowel disease. In some cases, the interval between the primary breast cancer and occurrence of colorectal lesions is so long that the critical history is lost or missed. Reported herein is a case of metastatic lobular carcinoma of the breast masquerading as a primary rectal cancer in a 62-year-old Japanese woman.

\footnotetext{
* Correspondence: hiros@hyo-med.ac.jp

'Department of Surgical Pathology, Hyogo College of Medicine, 1-1

Mukogawa-cho, Nishinomiya, Hyogo 663-8501, Japan

Full list of author information is available at the end of the article
}

\section{Case presentation}

A 62-year-old Japanese woman was referred to a nearby clinic because of progressive constipation for six months. Endoscopic examination of her colon revealed two circumferential strictures at the ascending colon and rectum (Figure 1a). Hematoxylin and eosin (HE) image of the endoscopic biopsy of the rectal lesion led us to a diagnosis of poorly differentiated adenocarcinoma of the rectum (Figure 1b). The biopsy of the lesion of the ascending colon revealed colitis. Total resection of the tumor was predicted to be difficult due to size and infiltrative border of the tumor. Therefore, the patient was subjected to chemo-radiotherapy. After five courses of the chemoradiotherapy in total of 45 Gy of radiation plus tegafur gimeracil oteracil potassium (TS-1) and irinotecan (CPT11) as chemotherapy with minimal clinical response, a 


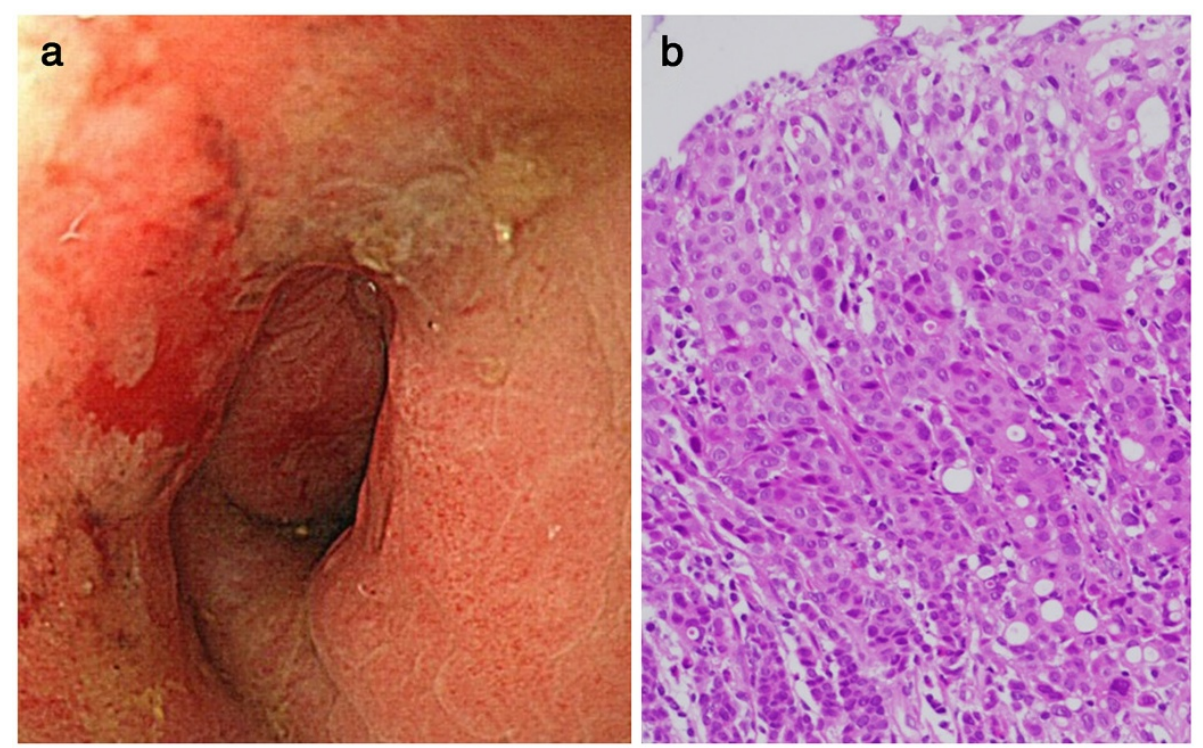

Figure 1 Preoperative endoscopy of the lesion. Endoscopy showed circumferential stricture with erosive change of the rectum (a), and biopsy specimen from the lesion revealed poorly differentiated adenocarcinoma (b) (HE stain, $\times 400$ ).

proctectomy was performed with low anterior resection. Macroscopically, the rectal mass was a diffusely invasive lesion with no clear border. Cut surface showed white trabeculae penetrating vertically through the muscularis propria (Figure 2).

Microscopic examination of resected specimen revealed a diffuse and infiltrative proliferation of small tumor cells (Figure 3a,b). There was no apparent degeneration or necrosis of the tumor cells, suggesting the minimal pathological effect of chemo-radiotherapy on the tumor cells. Minimal cohesion was observed among the tumor cells. Of note was the single-file arrangement of the tumor cells (Figure 3b), characteristically observed in lobular carcinoma of the breast, but rarely found in colon cancer. These observations of an HE-stained specimen made us suspect metastatic lobular carcinoma of the breast, instead of primary rectal cancer.

To confirm this, immunohistochemical analysis was performed (Figure 3c-3f). The tumor cells were positively

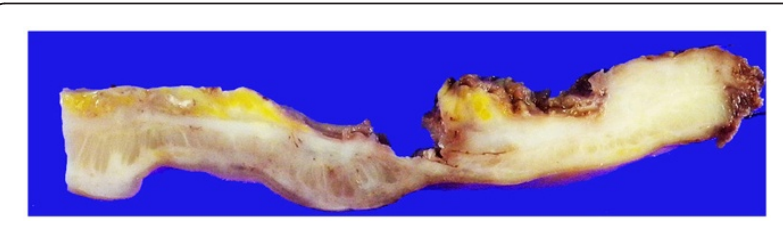

Figure 2 Cut surface of the resected rectal tumor after formalin fixation. Macroscopic examination showed a diffusely invasive lesion (in white) with no clear border. Note white trabeculae of the tumor penetrating vertically through the muscularis propria. The upper side was luminal whereas the lower side was serosal. stained for CK7 (Figure 3c) and Estrogen receptor $\alpha$ (Figure 3e), while they were negative for CK20 (Figure 3d) and E-cadherin (Figure 3f). In contrast, the background colon epithelial cells were positive for CK20 (Figure 3d) and E-cadherin (Figure 3f), while they were negative for CK7 (Figure 3c) and estrogen receptor $\alpha$ (Figure 3e).

We reexamined the patient's history and found that she had undergone surgery for left breast cancer 24 years earlier. The pathological diagnosis of the cancer was found to be 'poorly differentiated adenocarcinoma, scirrhous type, with metastasis in her left axillary lymph nodes. Unfortunately, the glass slides and the paraffin blocks of the breast cancer were lost during the turmoil of the Hanshin-Awaji Earthquake in 1995. Therefore, we could not reconfirm the diagnosis of the primary breast cancer.

We reexamined the endoscopic biopsy of the rectum (Figure 1b) immunohistochemically using the same set of the antibodies used for the resected specimen. The immunophenotype of the rectal biopsy was identical to that of the resected tumor (data not shown). Reexamination and comparison with this biopsy sample revealed that the tumor cells in the resected specimen appeared less cohesive than those in the rectal biopsy. This is probably due to the effect of the chemo-radiation therapy on the tumor cells before surgical resection.

Mammography, ultrasonography, and ${ }^{18} \mathrm{~F}$-fluorodeoxyglucose-positron emission tomography (FDG-PET)/ computed tomography $(\mathrm{CT})$ examination revealed no evidence of cancer in her remaining right breast and a local recurrence of the left breast cancer (data not 


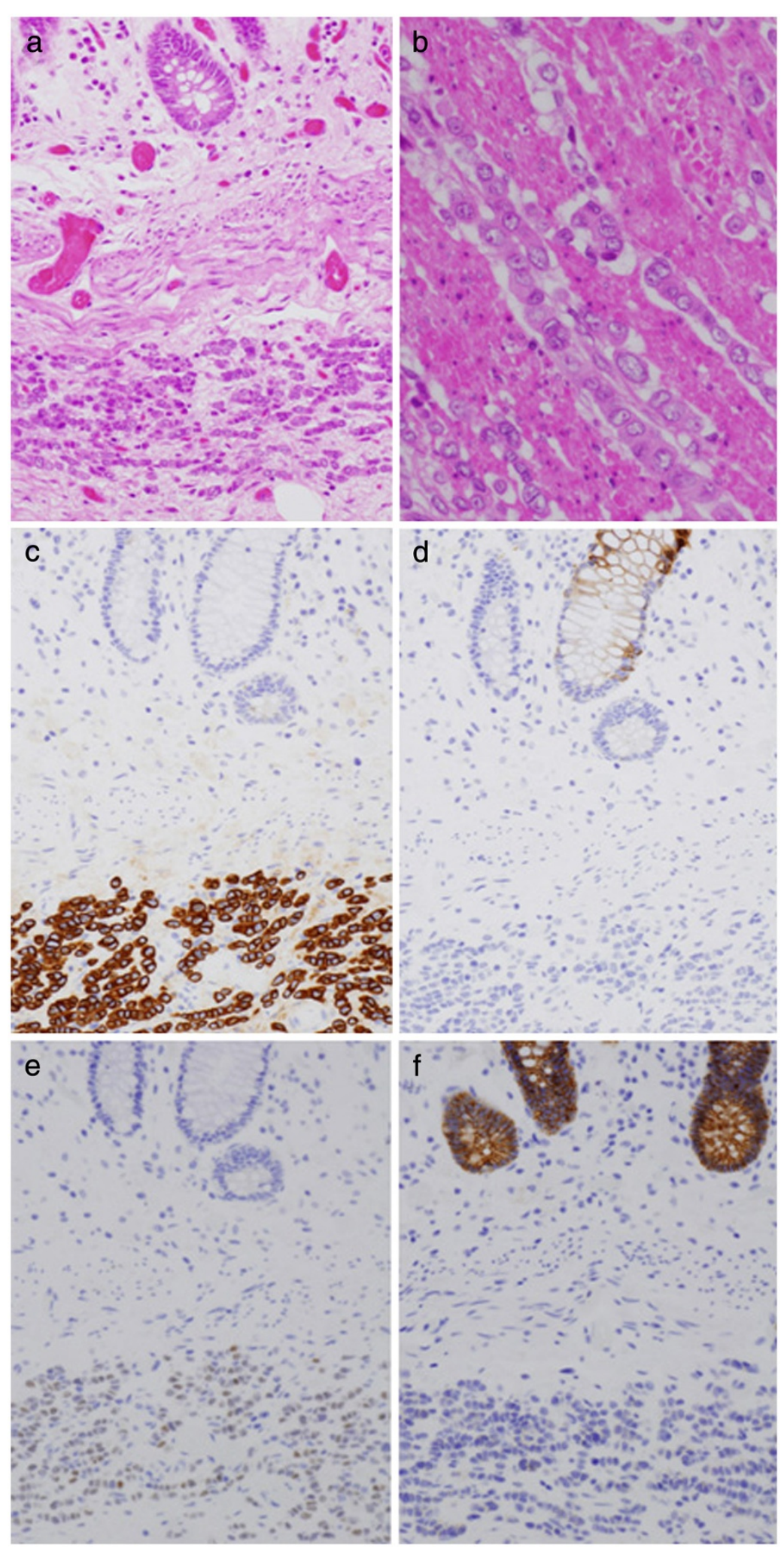

Figure 3 (See legend on next page.) 
(See figure on previous page.)

Figure 3 Histological examination of the resected rectal tumor. (a,b) Hematoxylin and eosin (HE) staining showed diffuse and infiltrative proliferation of small tumor cells in the submucosal region $(\mathbf{a}$, original magnification $\times 200)$ and through the muscularis propria $(\mathbf{b}$, original magnification $\times 400$ ). Minimal cohesion was observed among the tumor cells, and each tumor cell had an eccentric nucleus and eosinophilic cytoplasm. (c-f) Immunohistochemical findings. The tumor cells were positive for CK7 (c) but not for CK20 (d). They were positive for Estrogen receptor a (e) but negative for E-cadherin $(\mathbf{f})$. In $(\mathbf{a})$ and $(\mathbf{c}-\mathbf{f})$, the lower half of each figure was occupied by the tumor cell nests in the submucosal region, whereas in the upper half, the background normal colon crypts were observed (original magnification $\times 200$ ).

shown). There were two hot spots at the rectum and thyroid on FDG-PET/CT examination (data not shown). The former was considered to result from radiation therapy and/or proctectomy procedure, and the latter was presumably due to chronic thyroiditis.

Although we could not reconfirm the diagnosis of the primary breast cancer, the results described above strongly support our final diagnosis of rectal metastasis from lobular breast carcinoma.

\section{Discussion}

In this paper, we reported a case of rectal metastasis from lobular breast carcinoma, masquerading as a primary rectal cancer. The correct diagnosis was obtained by histopathological examination of the resected rectal mass after chemo-radiotherapy.

To our knowledge, at least 21 cases have been reported on metastatic lobular carcinoma of the breast to the colorectum [1-21]. There have been four reviews on the clinical or radiological spectrum of metastatic lobular carcinoma of the breast to the colorectum [22-25]. McLemore et al. reported that cases of gastrointestinal metastasis from primary breast cancer were as rare as 73 cases among 12,001 cases [22]. Among them, cases with colorectal metastasis were only 24 . As histological subtypes, lobular carcinoma of the breast predominates in comparison to ductal carcinoma [22,23].

In our case, history of primary breast cancer was unknown in the initial presentation of the colorectal lesion; the history was revealed after histopathological examination of the resected specimen. Among the 21 cases reported of metastatic lobular carcinoma of the breast to the colorectum, only one case was similar to our case [1]. In two cases, breast cancer and presumed metastasis were found coincidentally $[2,3]$. In the majority of the cases, a history of primary breast cancer was known in the initial presentation of gastrointestinal lesions and it was not difficult to suspect those lesions as relapse or metastasis of the breast cancer [4-14]. In one case, history of primary breast cancer was known in the initial presentation of the gastrointestinal lesions, but correct diagnosis could not be reached [15]. Harsløf et al. reported a case compatible with metastasis of lobular carcinoma of the breast to the colon although the primary breast cancer had not been identified [16].
There are a couple of reasons for difficulties in distinguishing metastatic lobular carcinoma of the breast to the colorectum from the primary colorectal cancer. First, the interval between the primary breast cancer and its metastatic relapse tends to be long. Therefore, a key to the correct diagnosis is recognition of a patient's history of breast cancer. Schwarz et al. reported that median interval between breast cancer and gastrointestinal metastasis was 6 years (range 0.25 to 12.5 years) [24]. McLemore et al. reported that mean interval between breast cancer and gastrointestinal metastasis was 7 years [22]. Among all the cases reported in the literature, 25 years was the longest interval between the diagnosis of the primary breast lobular carcinoma and the recognition of its metastasis to the colorectum [4]. Mistrangelo et al. reported a case for sigmoid colon metastasis from the primary lobular carcinoma of the breast 25 years before [4]. However, in that case, there was an episode of bone metastasis of the breast cancer 12 years before the colon metastasis [4]. In contrast, in our case, the 24-year interval was the second longest one and was diseasefree. This suggests that a long-term, close follow-up as well as recognition of patients' history is required for correct diagnosis of rectal metastasis from lobular breast carcinoma.

Secondly, metastatic lobular carcinoma of the breast to the colorectum presents endoscopic and radiologic appearance of linitis plastica-type lesion with circumferential stricture and wall thickening of the colorectum $[1,25]$. Arrangoiz et al. reported a case in which only pediatric colonscope could pass the narrowed lumen of the lesion [1]. Colorectal metastasis of lobular breast carcinoma is sometimes accompanied by nodular and cobble stone-like thickening of mucosa [5]. These macroscopic characteristics will present impression of the lesion as a poorly differentiated carcinoma as is often seen in the stomach. When the metastasis occurs around the terminal ileum lesion, a misdiagnosis may be made as an inflammatory bowel disease as Crohn's disease $[6,7,15]$. In fact, Calafat et al. reported metastasis of lobular carcinoma of the breast to ileum-colon, mimicking inflammatory bowel disease such as Crohn's disease [6]. Koos et al. reported a case where multiple metastases simulating Crohn's disease were found radiologically and intra-operatively in the colon and small bowel [7]. When metastatic cancer remains in submucosa, it will be difficult to obtain enough biopsy 
samples endoscopically, further strengthening the impression that the lesion may be non-tumorous [15].

\section{Conclusions}

Poorly differentiated adenocarcinoma of the colorectum is rarer than that of the stomach. Linitis plastica-type cancer of the colorectum is also rarer than that of the stomach. A lesson from the present case is that before we conclude a linitis plastica-type cancer of poorly differentiated type as a primary colorectal cancer, it is critical to exclude the possibility of metastatic colorectal cancer.

\section{Consent}

Written informed consent was obtained from the patient for publication of this Case report and any accompanying images. A copy of the written consent is available for review with the Editor-in-Chief of this journal.

\section{Abbreviations}

CT: Computed tomography; FDG-PET: ${ }^{18} \mathrm{~F}$-fluorodeoxyglucose-positron emission tomography; HE: Hematoxylin and eosin.

\section{Competing interests}

The authors declare that they have no competing interests.

\section{Authors' contributions}

IM and SH participated in the pathological final diagnosis of the case and prepared and edited the manuscript. NA performed the preoperative endoscopic examination. NM, MH, DY, TK, KT, TY, MN, HI, and NT were responsible for the chemo-radiotherapy, operations, and follow-up of the patient and helped IM and $\mathrm{SH}$ in preparation of the manuscript. All authors read and approved the final manuscript.

\section{Author details}

'Department of Surgical Pathology, Hyogo College of Medicine, 1-1 Mukogawa-cho, Nishinomiya, Hyogo 663-8501, Japan. ²Department of Surgery, Hyogo College of Medicine, Nishinomiya 663-8501, Japan. ${ }^{3}$ Aoyama Clinic, Kobe 650-0015, Japan.

Received: 10 July 2012 Accepted: 18 October 2012

Published: 31 October 2012

\section{References}

1. Arrangoiz R, Papavasiliou P, Dushkin H, Farma JM: Case report and literature review: metastatic lobular carcinoma of the breast an unusual presentation. Int J Surg Case Rep 2011, 2:301-305.

2. Razzetta F, Tassara E, Saro F, Sironi M, D'Ambrosio G: Rare abdominal metastases from occult lobular breast cancer: report of two cases. Updates Surg 2011, 63:129-133.

3. Rosati G, Ferrara D, Scarano E, Siciliano P: Colon and muscle metastases from lobular breast carcinoma: a very rare entity. Breast J 2012, 18:77-79.

4. Mistrangelo M, Cassoni P, Mistrangelo M, Castellano I, Codognotto E, Sapino A, Lamanna G, Cravero F, Bianco L, Fora G, Sandrucci S: Obstructive colon metastases from lobular breast cancer: report of a case and review of the literature. Tumori 2011, 97:800-804.

5. Malhotra A, Guturu P, Basim MS, Raju GS: A rare case of breast cancer metastasis presenting as linitis plastica of the stomach and colon (with videos). Gastrointest Endosc 2009, 70:552-553.

6. Calafat $P$, de Diller AB, Sanchez C: Breast carcinoma metastasis in ileumcolon and gallbladder simulating inflammatory diseases. Rev Fac Cien Med Univ Nac Cordoba 1999, 56:123-127.

7. Koos L, Field RE: Metastatic carcinoma of breast simulating Crohn's disease. Int Surg 1980, 65:359-362.

8. de Lima DC A, Alberti LR: Breast cancer metastasis to the colon. Endoscopy 2011, 43:E143-E144
9. Bustamante-Balén M, Navarro-Hervás M, Cuevas JM, García-Díez JV: Colon metastasis of a lobular breast carcinoma. Rev Esp Enferm Dig 2008, 100:249-250.

10. Landi F, Martí Gallostra M, Espin Basany E, Landolfi S, Armengol Carrasco M: Colon metastasis of lobular breast carcinoma. Cir Esp 2012, 90:470-471.

11. López Deogracias M, Flores Jaime L, Arias-Camisón I, Zamacola I, Murillo Guibert J, Suescun García R, Querejeta Usabiaga J, Martínez García F: Rectal metastasis from lobular breast carcinoma 15 years after primary diagnosis. Clin Transl Oncol 2010, 12:150-153.

12. Martínez Lesquereux L, Paredes Cotoré JP, Ladra González MJ, Beiras Torrado A: Colon metastasis of lobular breast cancer. Cir Esp 2010, 88:122-124

13. Okido M, Seo M, Hamada $Y$, Kurihara S, Matsumoto K, Konomi H, Kato M Metastatic breast carcinoma simulating linitis plastica of the colon: report of a case. Surg Today 2011, 41:542-545.

14. Signorelli C, Pomponi-Formiconi D, Nelli F, Pollera CF: Single colon metastasis from breast cancer: a clinical case report. Tumori 2005 91:424-427

15. Szabó J, Falkus B, Simon E, Brünner S, Baranyay F: Late gastrointestina metastases of invasive lobular breast carcinoma mimicking Crohn's disease. Orv Hetil 2010, 151:1666-1671.

16. Harsløf SS, Andersen LM, Hoyer U, Christiansen JJ: Breast cancer metastasis to the colon. Ugeskr Laeger 2010, 172:2309-2310.

17. Balja MP, Vrdoljak DV, Stanec M, Sitić S, Supić DK, Knezević F: Rectal metastasis from lobular carcinoma of the breast: a case report. Coll Antropol 2010, 34:719-721

18. Cervi G, Vettoretto N, Vinco A, Cervi E, Villanacci V, Grigolato P, Giulini SM: Rectal localization of metastatic lobular breast cancer: report of a case. Dis Colon Rectum 2001, 44:453-455.

19. Hernández V, Flor-Lorente B, Burgués O, Flor-Civera B, Oliver V: Anasarca as presentation of lobular breast carcinoma. Gastroenterol Hepatol 2000 23:338-340

20. Rabau MY, Alon RJ, Werbin N, Yossipov Y: Colonic metastases from lobular carcinoma of the breast. Report of a case. Dis Colon Rectum 1988, 31:401-402

21. Voravud N, el-Naggar AK, Balch CM, Theriault RL: Metastatic lobular breast carcinoma simulating primary colon cancer. Am J Clin Oncol 1992 15:365-369

22. McLemore EC, Pockaj BA, Reynolds C, Gray RJ, Hernandez JL, Grant CS, Donohue $\mathrm{JH}$ : Breast cancer: presentation and intervention in women with gastrointestinal metastasis and carcinomatosis. Ann Surg Oncol 2005, 12:886-894

23. Taal BG, den Hartog Jager FC, Steinmetz R, Peterse $H$ : The spectrum of gastrointestinal metastases of breast carcinoma: II. The colon and rectum. Gastrointest Endosc 1992, 38:136-141.

24. Schwarz RE, Klimstra DS, Turnbull AD: Metastatic breast cance masquerading as gastrointestinal primary. Am J Gastroenterol 1998 93:111-114.

25. Winston CB, Hadar O, Teitcher JB, Caravelli JF, Sklarin NT, Panicek DM, Liberman L: Metastatic lobular carcinoma of the breast: patterns of spread in the chest, abdomen, and pelvis on CT. AJR Am J Roentgenol 2000, 175:795-800.

doi:10.1186/1477-7819-10-231

Cite this article as: Matsuda et al: Metastatic lobular carcinoma of the breast masquerading as a primary rectal cancer. World Journal of Surgical Oncology 2012 10:231. 